\title{
Comparison of Optical VCSEL Models on the Simulation of Oxide-Confined Devices
}

\author{
Peter Bienstman, Roel Baets, Josip Vukusic, Anders Larsson, Michael J. Noble, Marcel Brunner, Karlheinz Gulden, \\ Pierluigi Debernardi, Laura Fratta, Gian Paolo Bava, Hans Wenzel, Benjamin Klein, Olaf Conradi, \\ Reinhold Pregla, Fellow, IEEE, Spilios A. Riyopoulos, Jean-François P. Seurin, Student Member, IEEE, and \\ Shun Lien Chuang
}

\begin{abstract}
We compare the results of different optical verticalcavity surface-emitting laser models on the position-dependent effects of thin oxide apertures. Both scalar and vectorial models as well as hybrid models are considered. Physical quantities that are compared are resonance wavelength, threshold material gain, and modal stability. For large device diameters and low-order modes, the agreement between the different models is quite good. Larger differences occur when considering smaller devices and higher order modes. It is also observed that the spread in the resonance wavelengths is smaller than that for the threshold material gain.
\end{abstract}

Index Terms-Distributed Bragg reflector lasers, laser modes, semiconductor device modeling, semiconductor lasers, surfaceemitting lasers.

\section{INTRODUCTION}

$\mathbf{I}$ N RECENT YEARS, the characteristics of vertical-cavity surface-emitting lasers (VCSELs) have improved enormously, especially after the introduction of oxidized apertures

Manuscript received March 21, 2001; revised August 17, 2001. The work of P. Bienstman was supported by the Flemish National Fund for Scientific Research (FWO-Vlaanderen) through a doctoral fellowship and also by the Belgian DWTC project IUAP IV-13. The work of B. Klein was supported by the National Science Foundation through the DesCArtES Center. The work of J.-F. Seurin and S. L. Chuang was supported by the Center for Computational Electromagnetics at the University of Illinois at Urbana-Champaign. The work at the University of Torino was carried out in the framework of an Italian National Project (MADESS II) and VISTA (VCSELs for Information Society Technology Applications). The work at Chalmers University was supported by the Swedish Foundation for Strategic Research (SSF). This work was supported by the European COST 268 action ("Wavelength scale photonic components for telecommunications").

P. Bienstman and R. Baets are with the Department of Information Technology, Ghent University/IMEC, B-9000 Ghent, Belgium (e-mail: Peter.Bienstman@rug.ac.be).

J. Vukusic and A. Larsson are with the Microtechnology Center at Chalmers (MC2) and the Photonics Laboratory, Department of Microelectronics, Chalmers University of Technology, SE-41296 Göteborg, Sweden.

M. J. Noble is with the U.S. Air Force, Research Lab Sensors Directorate, AFRL, SNHC, Hanscom AFB, MA 01731 USA.

M. Brunner and K. Gulden are with Avalon Photonics Ltd., CH-8048 Zurich, Switzerland.

P. Debernardi, L. Fratta, and G. P. Bava are with the IRITI-CNR, Dipartimento di Elettronica and INFM Torino, Politecnico di Torino, 10129 Torino, Italy.

$\mathrm{H}$. Wenzel is with the Ferdinand-Braun-Institut für Höchstfrequenztechnik, D-12489 Berlin, Germany.

B. Klein is with the Beckman Institute, University of Illinois, Urbana, IL 61801 USA.

O. Conradi and R. Pregla are with the FernUniversität Hagen, D-58084 Hagen, Germany.

S. A. Riyopoulos is with the SAIC, McLean, VA 22102 USA

J.-F. P. Seurin is with the Department of Electrical and Computer Engineering, University of Illinois, Urbana, IL 61801 USA and also with Princeton Optronics, Princeton, NJ 08543-8627 USA.

S. L. Chuang is with the Department of Electrical and Computer Engineering, University of Illinois, Urbana, IL 61801 USA.

Publisher Item Identifier S 0018-9197(01)10250-2. to realize electrical and optical confinement. Today's VCSELs show low-threshold current densities [1] and high output powers [2]. Moreover, their circular beam profiles and the possibility to integrate then into 2-D arrays [3] make them suitable candidates for short-range optical communications and optical interconnects.

In order to design the next generation of VCSELs with even better performance, it is imperative to be able to model the optical, electrical, and thermal effects that come into play in these devices. Specifically, the modeling of the optical field is quite challenging, since the Helmholtz equation is not separable in this case. Moreover, these structures can have large index contrasts, especially so for oxide-confined and airpost VCSELs. Over the years, a number of approaches have been proposed to model VCSELs, either scalar or vectorial, or approximate or rigorous. However, comparing these models and therefore validating their underlying assumptions has been difficult, if not impossible, since the published results obtained with these models are all for different VCSEL structures. It is the aim of the present paper to compare many of today's important VCSEL models on the same benchmark problem, thereby quantifying the spread of results among the different models.

The rest of this paper is organized as follows. Section II gives a brief overview of each of the models involved in this comparison. The models are subdivided into scalar models, models with both a scalar and a vectorial implementation, hybrid scalar-vectorial models, and finally vectorial models. The names in parentheses occurring in each paragraph title will be used to refer to the results of that particular model. These names usually derive from an acronym or from the name of a university or company. The benchmark structure itself will be described in Section III. Subsequently, the simulation results of the models will be compared in Section IV. Finally, Section V summarizes our main conclusions. The numerical results of the simulations will be given in the Appendix, to facilitate comparison with future models.

\section{OPTICAL VCSEL MODELS}

\section{A. Scalar Models}

This class of models starts out from the scalar Helmholtz equation. In doing so, all polarization information is lost. This is usually not a problem, except when considering fields that have a relatively large transverse wavevector component, as is the case in higher order modes or smaller devices. The main advantage of these models is obviously their speed, making them 
suitable design tools when the focus is more on capturing device trends rather than on obtaining numerically accurate results.

Hadley's Effective Index (EI) Model: In this model, implemented among others at Avalon Photonics, the lateral optical waveguide in the VCSEL is approximated by an EI step located at the radius of the oxide aperture. The index of the waveguide core is calculated from a field-weighted average of the different refractive indices in the VCSEL layer structure [4]. The index step to the cladding region is found by multiplying the core index with the relative wavelength shift of the cavity mode when the structure is oxidized $\left(\Delta n=n_{\text {core }} \cdot \Delta \lambda / \lambda\right)$ [4]. The optical modes are linearly polarized (LP) modes, as found from the standard solution of a step index waveguide.

EI Method Using Eigenmodes (Chalmers): This model implemented at Chalmers University is also based on Hadley's EI model [5] for which the longitudinal field distribution is calculated by solving a 1-D eigenvalue equation. The transverse field distribution was obtained by solving

$$
\frac{\partial E_{\text {transv }}}{\partial t}=\frac{i c}{2 k_{0}\left\langle\varepsilon_{i}\right\rangle}\left(\frac{1}{r} \frac{\partial}{\partial r} r \frac{\partial}{\partial r}-\frac{m^{2}}{r^{2}}+k_{0}^{2} \Delta \varepsilon_{\text {eff }}\right) E_{\text {transv }}
$$

where $E_{\text {transv }}$ was assumed to have a time dependence according to

$$
E_{\text {transv }}=E_{m n}(r) e^{i \Delta \omega_{m n} t} .
$$

By inserting (2) into (1), we arrive at the following eigenvalue equation:

$$
A_{m} E_{m n}=\Delta \omega_{m n} E_{m n} .
$$

We can gather from (2) that the real and complex parts of the eigenvalue $\Delta \omega_{m n}$ are closely related to the modal cavity resonance and gain, respectively.

Prior to numerical calculations, the operator $A_{m}$ in (3) was discretized uniformly by 100 points on a $10-\mu \mathrm{m}$ mesh window. To calculate a mode profile with an accompanying eigenvalue took $\sim 1$ s. Calculations were done on a 333-MHz Pentium-II $\mathrm{PC}$ in a Matlab environment.

Effective Frequency (EF) Model: This model is also based on Hadley's EI model [4]-[6]. A comprehensive description can be found in [7]. The main differences to Hadley's model can be summarized as follows.

1) The optical frequency $\omega$ is considered to be a complex number. Its real and imaginary parts determine the modal wavelength and the decay constants, respectively, of the eigenmodes of the device.

2) The term $\omega^{2} \varepsilon(\boldsymbol{r}, \omega)$ occurring in the scalar wave equation is linearized around a real valued reference frequency $\omega_{0}$. For the device considered here, $\omega_{0}$ was chosen to correspond to a vacuum wavelength of $980 \mathrm{~nm}$.

3) Because the dielectric function $\varepsilon(\boldsymbol{r}, \omega)$ may depend on the frequency $\omega$, temporal dispersion is included.

4) Instead of having to solve a time-dependent, in-plane partial differential equation as in [4]-[6], we have to determine a complex valued eigenvalue $\nu$ of a time-independent ordinary differential equation, which gives the resonance wavelengths and decay constants of the modes of the whole resonator. The complex valued eigenvalue $\nu$ is proportional to the frequency difference $\omega_{0}-\omega$.

5) The derivation of the in-plane equation is based upon a variational principle by minimizing a functional.

By comparing with other models, it should be also noted that, in both the vertical direction (perpendicular to the epitaxial layers) and the in-plane direction (parallel to the layers), the simulation domain is treated as infinitely extended, and exact transparent boundary conditions are used. Additionally, continuity of both the electric field strength and its normal derivative is required at all interfaces between different materials.

The numerical procedure of locating the material gain where the imaginary part of $\nu$ vanishes takes about $1 \mathrm{~s}$ on a $667-\mathrm{MHz}$ DEC-Alpha workstation, for the benchmark structure of Section III. The linearization with respect to frequency leads to slight deviations of the results from the exact value, even for a purely planar configuration.

Optimized-Waist Paraxial Eigenmodes Using a Cavity $S$-Matrix (PREVEU): In the Paraxial Radiation Eigenmodes for VCSEL Emulation (PREVEU) model developed at Science Applications International Corporation [8], [9], the cavity round-trip S-matrix is obtained analytically using a paraxial mode expansion with the mode waist as a free parameter. No longitudinal index-averaging, or separable approximations, are assumed. Distributed Bragg reflectors (DBRs) are modeled by equivalent hard mirrors, located at the effective phase penetration and the effective diffraction lengths, respectively, for the standing-wave condition and reflected wavefront computation. Wavefront "clipping" due to finite mirror radius, and gain guiding due to finite gain area, are evaluated analytically in terms of nondiagonal S-matrices. In treating apertures, losses from wide-angle scattering outside the paraxial propagation cone are computed, applying the Born approximation to the EM scattering theory. The aperture-induced phase-shift is computed by Schrödinger perturbation theory. Scattering losses overtake combined losses from all other factors at small apertures. Wave propagation between gain, aperture, and mirror elements is modeled by the uniform medium paraxial propagator, yielding simply a rescaling of the paraxial beam parameters with distance traveled. Finally, the diffracted and deformed wavefront is projected onto the original, obtaining the analytic expression for the round-trip matrix in terms of current aperture, mode waist, and cavity Fresnel number. Variation of the round trip eigenvalue with respect to the mode waist yields the mode structure analytically.

Execution time for obtaining the first 20 modes is less than $100 \mathrm{~ms}$ on a Pentium 500 machine. Computed results yielded excellent agreement with near-field profile measurements on a 780-nm etched mesa device.

\section{B. Models With Scalar and Vectorial Implementation}

These models can be either formulated in a scalar or vectorial way and are, therefore, potentially well suited to evaluate the necessity of a vectorial implementation.

Coupled Mode Model (Torino): In this model [10], [11], implemented at the Politecnico di Torino, Italy, the vectorial electromagnetic field is expanded on the continuous basis of the 
TE and TM free space modes of a reference medium in cylindrical coordinates. These are expressed in terms of Bessel and trigonometric functions and are labeled by the continuous transverse wavevector $k$, and by the discrete azimuthal index $m$. In each longitudinally homogeneous layer of the device, the coupling between the expansion modes is determined by the perturbation to the reference refractive index induced by the device transverse structure. Through the formal solution of the coupled-mode equations, it is possible to define a relation between the electric field at any two positions of the resonator. The boundary conditions are set by the self-consistency between backward and forward waves at two arbitrary sections and define the threshold condition of each mode as the solution of an eigenvalue problem: eigenvalues are related to threshold gains and lasing frequencies of the modes, while the corresponding eigenvectors give the expansion coefficients and allow the reconstruction of the fields. The only approximation in this approach is the discretization of the continuous wavevector $k$. The adopted discretization step and the number of modes $(k, m)$ included in the mode expansion fix the dimensions of the eigenvalue problem. Different azimuthal modes are coupled only in the case of noncircularly symmetric structures. We have implemented this model with Matlab on a Pentium $400 \mathrm{MHz}$ PC and finding a laser mode for the benchmark structure takes about 4 min.

The same formalism can be applied by adopting a simplified scalar basis for the field expansion that can be derived from the vectorial basis in the TEM limit. In this way, a comparison between the vectorial treatment and the LP scalar approximation can be carried out and the range of validity of the scalar approach can be discussed. The size of the LP problem is reduced by a factor of 2 (TEM instead of TE and TM modes) which corresponds to a factor of 4 decrease in computational time.

Numerical Mode-Matching Method (UI-NMM): To find the vector modes of the VCSEL cavity, a numerical mode-matching method (NMM) [12] is used. The starting point of our method is similar to the approach described in [13]: the VCSEL structure is placed in a perfect conducting cylindrical waveguide and the modes of that perfect conducting waveguide are used as an expansion basis. An orthonormal basis is calculated from this expansion basis using the modified Gram-Schmidt algorithm. The finite-difference operators are then projected onto a smaller subspace. The projected operators no longer contain the field finite-difference operators. At the same time, this method avoids the calculation of the overlap integrals between the field and refractive index profiles. Thus, our formulation is not structure-dependent (in the sense that it is not limited to structures with only single step-index profiles) and permits the accurate modeling of VCSEL structures with complex permittivities of arbitrary transverse profiles. Furthermore, Krylov subspace techniques are used to improve computation speed. An efficient algorithm to obtain the resonant wavelength, quality factor, and "hot-cavity" threshold gain for each mode is derived. The details of the optical model are given in [14]. This solver was used in conjunction with a comprehensive rate-equation solver to model complex VCSEL structures [15].

The computational resources to run this code are fairly modest: on an Intel Celeron $366 \mathrm{MHz}$ with $128 \mathrm{MB}$ of RAM, simulation times to find the characteristics of one mode (resonant wavelength, "hot-cavity" threshold gain) are on the order of a few seconds to a couple of minutes.

Using the NMM, a scalar formulation was also derived. It is observed that the results closely match the vector results, especially for the wavelength. The largest discrepancies were observed for small diameters $(d=1$ and $2 \mu \mathrm{m})$ when the aperture is at a standing-wave anti-node (position no. 5). In this case, the relative difference in wavelength values is still negligible but the relative difference in threshold gain values is $7.6 \%$ for $d=2 \mu \mathrm{m}$ and $5.2 \%$ for $d=1 \mu \mathrm{m}$. For most practical cases, the scalar version of our code is sufficient. Compared to the vector version, computation times are divided by eight and memory requirements are divided by four.

For the simulation results given in the rest of this paper, the vectorial version of the model was used.

\section{Hybrid Models}

These approaches combine both scalar and vectorial approaches to deal with different subproblems of the VCSEL model.

Weighted Index Method With Parasitic Mode Loss (WIMP): The Weighted Index Method with Parasitic mode loss (WIMP) is a semi-analytic, hybrid scalar-vector technique for computing the relevant optical mode data (e.g., wavelength, threshold gain,and confinement factor) of an oxide-apertured VCSEL [16]-[18]. The method is born out of a quasi-rigorous solution of Maxwell's equations for an azimuthally symmetric layered geometry. For each eigenmode of the VCSEL, the total electric and magnetic vector fields are segregated into two parts: a separable portion and a nonseparable portion. This is the crux of the method.

The separable fields are defined such that they exactly solve Maxwell's equations for an underlying separable VCSEL geometry. Both the separable fields and their associated separable VCSEL geometry are simultaneously computed using the Weighted Index Method (WIM). In practice, for the sake of efficiency, we make the "linearly polarized" mode approximation, thereby yielding a representation of the separable fields where only the dominant vector field component is considered. The explicit results of this calculation are, for each eigenmode: separable electric and magnetic fields, corresponding separable geometry, resonant wavelength, confinement factor, and threshold gain. Here, the threshold gain compensates for mirror and absorption loss, as well as the two optical loss mechanisms present in the separable geometry. Diffraction loss comes about due to the nonseparablity of the real geometry, which is captured by the second part of the WIMP.

The nonseparable vector fields solve a set of Maxwell's equations in the original VCSEL geometry but with an additional volumetric source current stemming from the separable fields. The volumetric source current depends on two things: the relative strength of the separable fields, and the difference between the separable geometry and the actual geometry. Physically, we may think of the nonseparable fields as being the fields radiated by the separable source fields to compensate for the "nonseparability" of the VCSEL geometry. This is a semi-analogous line of reasoning to Huygen's principle for wave propagation. 
We solve for the nonseparable fields by expanding them in a Fourier basis set in the longitudinal direction. In order to retain the full vector stature of these fields, we use two orthogonal basis sets: a transverse electric set and a transverse magnetic set. The strength of each Fourier component is solved exactly in closed form using standard electromagnetic techniques; the diffraction loss is computed concurrently. The explicit results of this calculation are, for each eigenmode, a Fourier representation of the nonseparable fields and the diffraction loss. Finally, the separable and nonseparable solutions are combined and the threshold gain is adjusted to account for all optical loss mechanisms: absorption, mirror, and diffraction.

\section{Vectorial Models}

It is the aim of these models to solve Maxwell's equation exactly, without resorting to any approximations other than a nonzero discretization length or a finite number of terms retained in a series expansion. Therefore, these models are slower than their scalar counterparts, but they are the only resort when studying polarization properties or when considering fields with a significant amount of nonparaxial propagation (higher order modes, small devices, etc.).

Method of Lines (MoL): The method of lines (MoL) has been used to solve the vectorial wave equation in cylindrical coordinates [19], [20]. The structure is divided into layers where the material parameters are constant in the axial direction. An arbitrary distribution of the permittivity in the radial direction is possible. The wave equation has been discretized in the radial direction and trigonometric functions have been introduced in the azimuthal direction. The refractive index can also be variable in the azimuthal direction. In this case, Fourier series can be used instead of one single trigonometric function [21].

In the direction of propagation, analytic solutions are considered. The relation between the electric and magnetic fields at the boundary of two layers can be expressed as a complex admittance. Then, the impedance/admittance transformation concept has been used to transform the admittance into a reference plane where an eigenvalue problem must be solved. The admittance transformation concept is an excellent method to analyze structures with several (hundreds!) of different layers. It is a stable procedure, which has been proven by several examples [22].

The optical gain is introduced as the imaginary part of the complex refractive index of the active region. The whole structure has been calculated for the condition that the imaginary part of the complex resonance frequency vanishes. After solving the eigenvalue problem, the wavelength of the resonator and the optical field can be calculated in the whole structure.

The modeling of the task presented in this paper (see Section III) took 15 min in Matlab on a Pentium II 333-MHz PC to calculate the wavelength and the optical gain of one mode.

Green's Function Model (Green): The Green's function approach is based upon a mode eigenvalue equation forcing the modal gain to equal the modal loss, thereby enforcing the semiclassical lasing condition for the modes. The eigenvalue is defined as the complex number multiplying the gain in order that the semiclassical lasing condition is satisfied [23]. The mode equation is formulated as an integral eigenvalue equation incorporating the tensor Green's function of the VCSEL structure. This is done to take advantage of the fact that the Green's function can handle open boundaries in a natural way. The eigenvalue equation is discretized using the method of moments, resulting in a small, dense matrix eigenvalue problem that is rapidly solved. The slow part of the problem is evaluating the Green's function matrix elements between source and receiver "bricks." These matrix elements are calculated by separating the VCSEL structure into planar and nonplanar parts, assuming that the underlying VCSEL structure is planar. The Green's function for the planar part is calculated by expanding the source and receiver in plane waves and propagating the resulting plane wave fields through the structure. The Green's function for the nonplanar part (e.g., the oxide aperture) is calculated by treating the nonplanar regions as effective volumetric polarization sources in the eigenvalue equation, making the eigenvalue equation into a generalized eigenvalue equation. This calculation is difficult, but it need only be performed once for a wide range of VCSEL structures and gain configurations, which was the goal of this effort. The simulations presented here took roughly an hour for each mode on a DEC Alpha 533-MHz processor, but the modes may be recalculated for changing gain conditions in a matter of seconds.

Eigenmode Expansion With PML (CAMFR): In this approach, the structure is divided into sections with a constant refractive index profile along the propagation axis, and the field in each of these sections is expanded onto the eigenmodes of that particular section. In order to get a discrete set of radiation modes, the VCSEL is placed in a perfectly conducting metal cylinder. This cylinder is coated with a perfectly matched layer (PML), so as to eliminate the parasitic reflections from it, thereby effectively simulating an open structure [24]-[26]. Thanks to these absorbing boundary conditions, the cylinder can be placed much closer to the VCSEL, leading to a smaller computational volume and an improved computation time. At the interfaces between different sections, mode matching is used to decompose the field into the eigenmodes of the new section. This procedure ultimately gives rise to scattering matrices describing the top and the bottom half of the cavity. These matrices can be used to express the condition that the lasing mode should have a round-trip gain of unity. Wavelength and material gain are varied to achieve this. The entire approach is implemented in our simulation tool CAMFR [26] ${ }^{1}$. The simulation results in this paper were obtained with a radius of the metal cylinder of $12-0.05 \mathrm{j} \mu \mathrm{m}$ and 200 modes. Locating a lasing mode takes on the order of $5-10 \min$ on a $250-\mathrm{MHz}$ Ultrasparc II.

\section{BENCHMARK STRUCTURE}

The benchmark structure originated from a modeling exercise [27] in the European COST268 action, which is an open framework to facilitate the free flow of scientific ideas and results. Participation to this modeling exercise was completely open, and in effect also many non-European groups collaborated. In

${ }^{1}$ Available: http://camfr.sourceforge.net. 


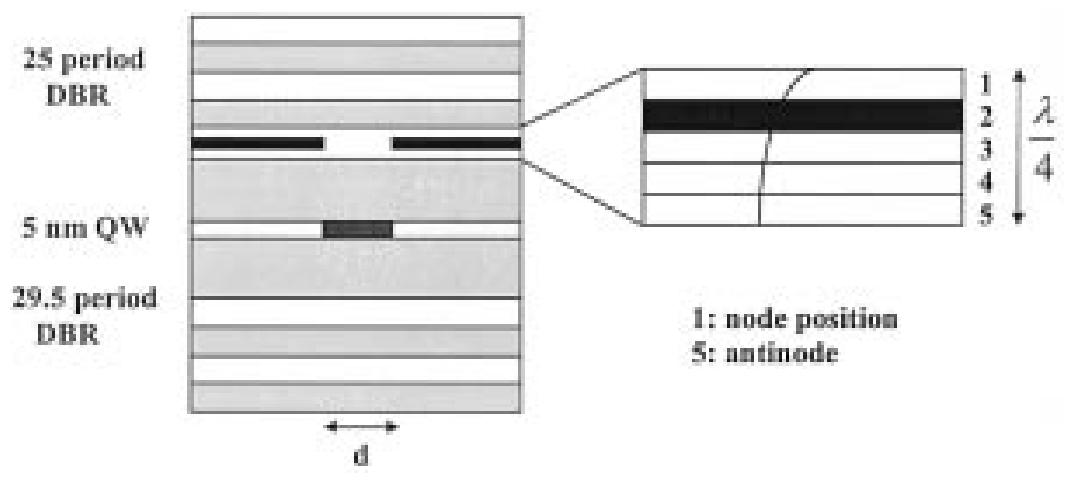

Fig. 1. Benchmark structure.

TABLE I

LAYER THICKNESSES AND REFRACTIVE INDICES OF BENCHMARK STRUCTURE

\begin{tabular}{|c|c|c|c|c|}
\hline & thickness & material & Index & \\
\hline air & & air & 1 & \\
\hline 24 pair & 69.49 & GaAs & 3.53 & \\
\hline DBR & 79.63 & AlGaAs & 3.08 & \\
\hline \multirow{5}{*}{$\begin{array}{c}\text { oxide } \\
\text { window }\end{array}$} & 69.49 & GaAs & 3.53 & \multirow{5}{*}{$\begin{array}{l}r<d / 2 \\
r>d / 2\end{array}$} \\
\hline & $63.71-x$ & AlGaAs & 3.08 & \\
\hline & 15.93 & AlAs & 2.95 & \\
\hline & & AlOx & 1.60 & \\
\hline & $x$ & AlGaAs & 3.08 & \\
\hline \multirow{4}{*}{$\begin{array}{c}\text { lambda } \\
\text { cavity }\end{array}$} & 136.49 & GaAs & 3.53 & \multirow{4}{*}{$\begin{array}{l}r<d / 2 \\
r>d / 2\end{array}$} \\
\hline & 5.00 & QW & $3.53+\mathrm{j} n_{i}$ & \\
\hline & & & $3.53-\mathrm{j} 0.01$ & \\
\hline & 136.49 & GaAs & 3.53 & \\
\hline 29.5 pair & 79.63 & AIGaAs & 3.08 & \\
\hline DBR & 69.49 & GaAs & 3.53 & \\
\hline substrate & & GaAs & 3.53 & \\
\hline
\end{tabular}

\begin{tabular}{c|c} 
Pos & $\boldsymbol{x}$ \\
\hline 1 & 63.71 \\
2 & 47.78 \\
3 & 31.85 \\
4 & 15.93 \\
5 & 0.00
\end{tabular}

$d=1,2,4,6,8 \mu \mathrm{m}$

the end, ten different groups participated in the comparison, resulting in a significant cross section of the currently pursued VCSEL modeling research.

The structure itself is detailed in Fig. 1 and Table I. It is an AlGaAs VCSEL designed for operation around $980 \mathrm{~nm}$. The bottom reflector consists of 29.5 pairs of AlGaAs-GaAs, while the top mirror has 25 pairs. In the lower $\lambda / 4$ layer of the top mirror, a $\lambda / 20$-thick oxide aperture is placed. The gain in the 5-nm-thick quantum qwell $(\mathrm{QW})$ is assumed to have a stepindex profile with the same dimensions as the oxide aperture. Outside of the aperture, the QW exhibits loss. The structure is grown on a GaAs substrate. The parameters that are varied in the simulation are the position of the aperture with respect to the optical field (from '1 - node position' to '5-antinode position' and the diameter of device (from 1 to $8 \mu \mathrm{m}$ ).
At this point, we want to point out that we do not include quantitative experimental verification for the modeling results. Experimental verification would not be as useful at it first seems, because a fabricated device will never have exactly the same geometry (layer thickness, material composition, etc.) as a proposed design, making exact quantitative comparisons awkward. Additionally, in real-life devices other aspects are at play, such as current flow and thermal effects, which are not captured by most of the models involved. We do not consider this to be a drawback of this paper, as it focuses solely on the optical aspects of VCSEL modeling, which is in itself already quite a broad and challenging topic.

\section{COMPARISON OF RESULTS}

Fig. 2 shows the resonance wavelength for the fundamental mode $\left(\mathrm{LP}_{01}\right.$ for the scalar modes, $\mathrm{HE}_{11}$ for the vectorial models) as a function of oxide position for a VCSEL with an $8-\mu \mathrm{m}$ diameter. The top curve corresponds to the planar VCSEL, i.e., the central region extending toward infinity. All the curves are blue-shifted with respect to the planar results, since the outer region has a lower EI.

Generally, the trends of the different models agree very well (within $0.5 \mathrm{~nm}$ ), which is to be expected for such a large structure. The PREVEU model, however, predicts a much smaller position-dependent resonance shift. Also note that the two curves for the scalar and vectorial version of the Torino model coincide. The same is true for the Chalmers and the EI model, since they are both variants of Hadley's EI model. It is also interesting to observe that there is no clear clustering between the scalar models (full lines) and the vectorial ones (dashed lines).

Fig. 3 shows the threshold material gain for the fundamental mode for the same $8-\mu \mathrm{m}$ VCSEL. When the oxide moves toward the field minimum, confinement is lost in this particular VCSEL design and the threshold material gain goes up. Apart from the PREVEU model, there is a tendency for the vectorial models to predict higher thresholds than the scalar models. This is illustrated further in Fig. 4, which shows the relative threshold difference normalized to the arbitrarily chosen EF model, which also shows the lowest threshold. First of all, we can see that the spread in threshold is significantly higher than the spread in wavelength results. To study the differences between scalar and vectorial approaches, we first have to distinguish between scalar 


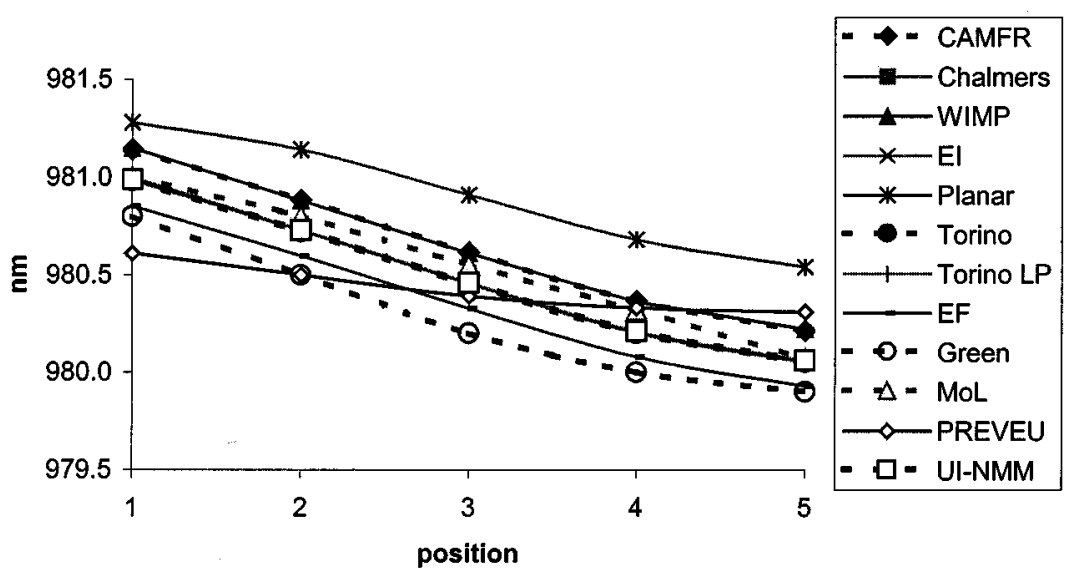

Fig. 2. Resonance wavelength for the fundamental mode as a function of oxide position for an 8- $\mu$ m-diameter VCSEL. (Full lines: scalar models. Dashed lines: vectorial models.)

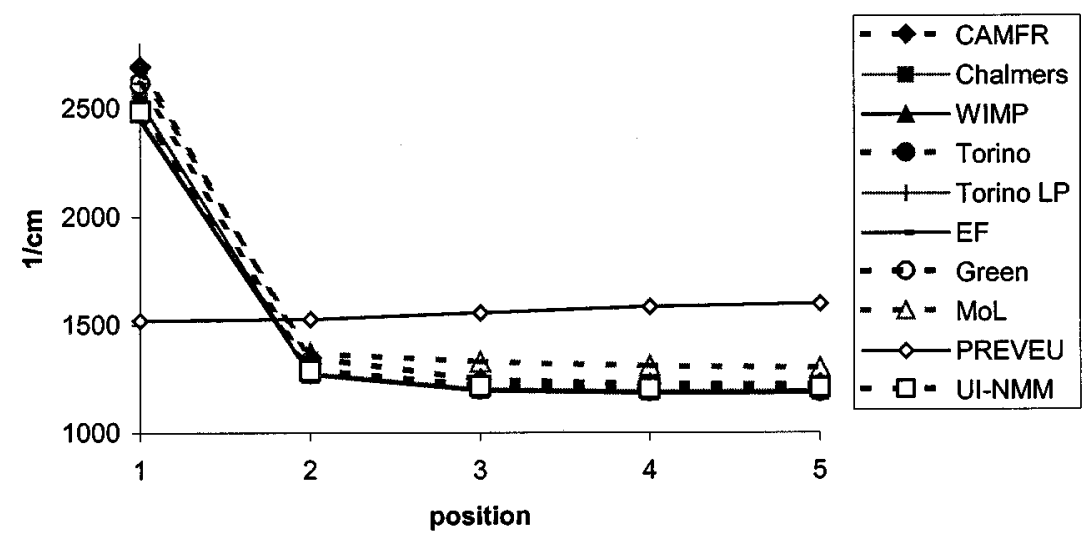

Fig. 3. Threshold material gain for the fundamental mode as a function of oxide position for an $8-\mu$ m-diameter VCSEL.

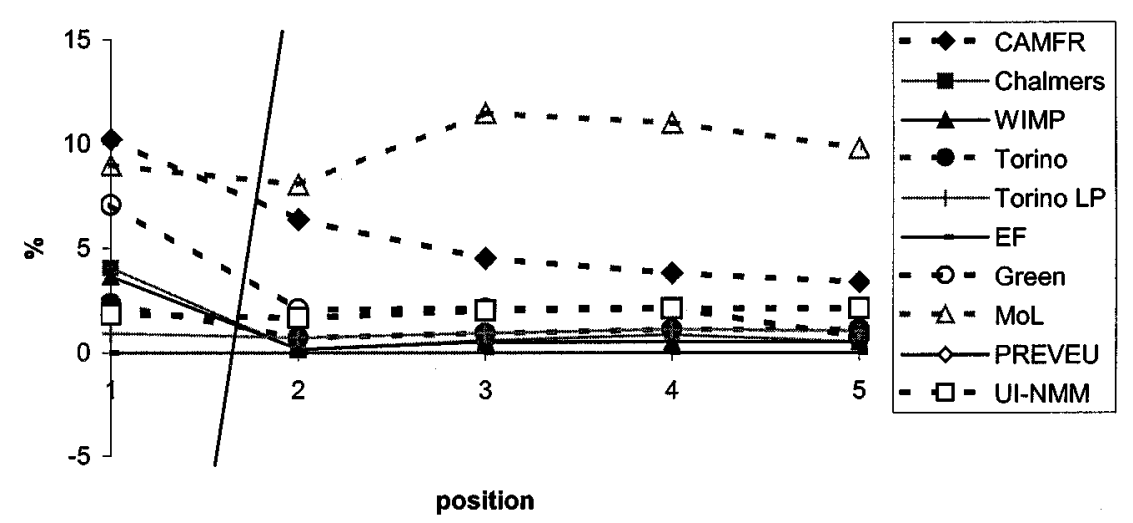

Fig. 4. Relative threshold material gain difference normalized to the EF model, for the fundamental mode as a function of oxide position for an 8- $\mu \mathrm{m}$-diameter VCSEL.

models that also imply a paraxial approximation (like the EI, Chalmers and EF models) and those that do not (WIMP, Torino LP, PREVEU, and the UI-NMM scalar version). The former are unable to take diffraction losses into account and therefore tend to predict lower thresholds, which can be seen from Fig. 4. The nonparaxial scalar models try to include these diffraction losses, but it is uncertain whether this is achieved as accurately as in a full vectorial model. Full-vectorial models (CAMFR, MoL, Green, Torino, and UI-NMM) take these diffraction losses fully into account, and therefore they typically predict higher thresh- olds than the other models (see the dashed lines in Fig. 4). However, it is to be noted that the spread among the vectorial models is significantly higher than the spread among the scalar models. We attribute this to the influence of the boundary conditions, which becomes more prominent as radiation caused by diffraction propagates to the numerical boundary and can cause parasitic reflections [25].

When considering the scalar and the vectorial implementation of the Torino model, it can be seen that the differences are relatively minor. The largest difference occurs at the node posi- 


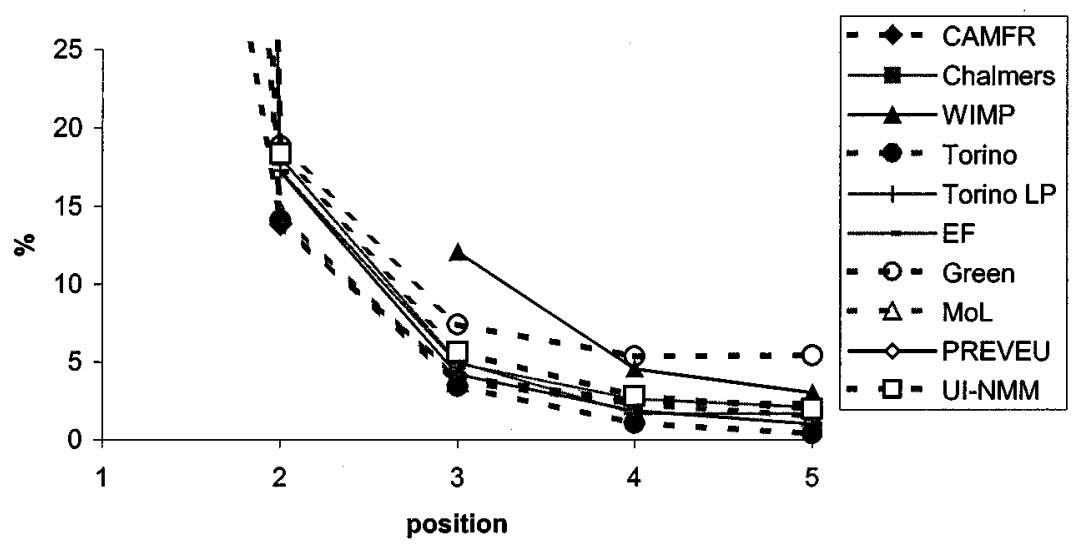

Fig. 5. Modal stability as a function of oxide position for an 8- $\mu$ m-diameter VCSEL.
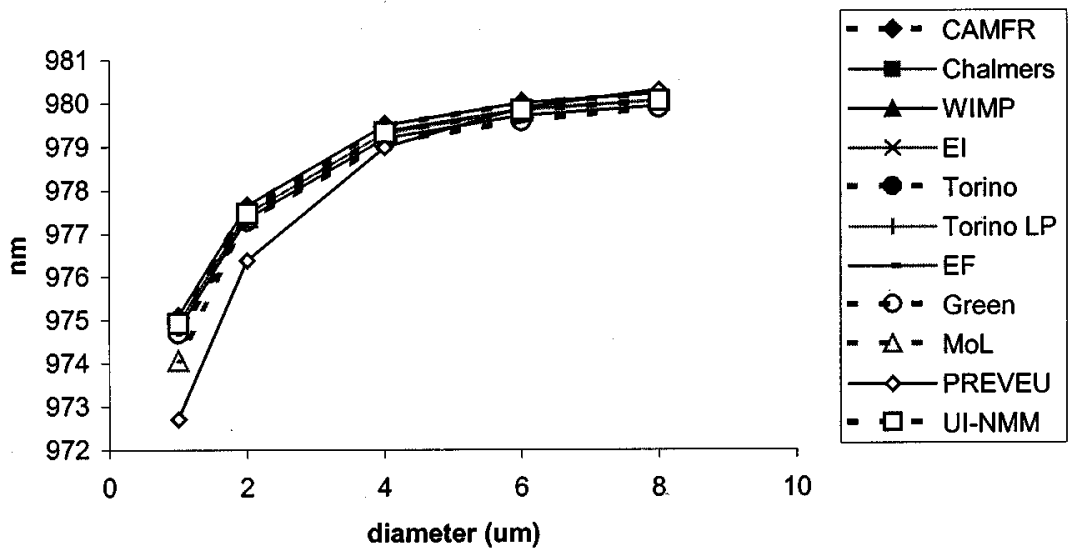

Fig. 6. Resonance wavelength for the fundamental mode as a function of diameter for an antinode oxide.

tion, where the vectorial version has a slightly higher threshold. These results seem to be typical for the Torino model, however. For the UI-NMM method, the difference between the scalar and the vectorial version is larger at the antinode position. These two models are the only nonparaxial scalar models that also have a vectorial version. Since comparing the differences between the scalar and the vectorial version gives different results for the different models, it would not be advisable to draw any firm conclusions on the ability of nonparaxial scalar models to accurately model diffraction loss.

Let us now consider simulation results on the first order mode. For scalar models, this is $\mathrm{LP}_{11}$. For vectorial models, we choose $\mathrm{TE}_{01}$. We could also have chosen $\mathrm{TM}_{01}$ or $\mathrm{HE}_{21}$, since all three of these modes degenerate to $\mathrm{LP}_{11}$ in the scalar limit, but as the difference between these three modes is rather small, we restricted our parameter space to $\mathrm{TE}_{01}$ only.

In Fig. 5, we plot the modal stability, as defined by the percentage difference in threshold between the higher order mode and the fundamental one, written as

\section{Threshold first order - Threshold fundamental Threshold fundamental}

For node oxides, the higher order mode suffers much more from the lack of confinement than the fundamental mode. This leads to an increased modal stability at the expense of a higher threshold for the fundamental mode. We also see that comparing modal stability leads to an even higher spread in results than when comparing threshold gains or resonance wavelengths.

After keeping the diameter fixed and varying the oxide position, we now keep the oxide at the antinode position and vary the diameter. From Fig. 6, we see a decrease in lasing wavelength for decreasing aperture size. To further clarify the spread between the models, we plot in Fig. 7 the differences in predicted wavelength with regard to the EF model. The spread between the models is mostly of the same order of magnitude as in Fig. 2, although this time some models start to deviate from the general trend in going to smaller apertures (most notably MoL and PREVEU). As for the other models, the CAMFR results coincide very well with the WIMP model, and the EI, Chalmers, Torino, and UI-NMM models also give very similar results.

In Fig. 8, we plot the relative wavelength difference for the first-order mode. This time, the spread between the models is significantly higher and also increases as we move toward smaller diameters. The MoL results predict the longest wavelengths. The WIMP results no longer coincide with the CAMFR results and predict much shorter wavelengths. In spite of this larger spread in results, there is still no clear clustering between scalar and vectorial results, a conclusion consistent with the previous resonance wavelength calculations.

It is also interesting to consider the wavelength difference $\lambda_{1}-\lambda_{0}$ between the first order and the fundamental mode. This 


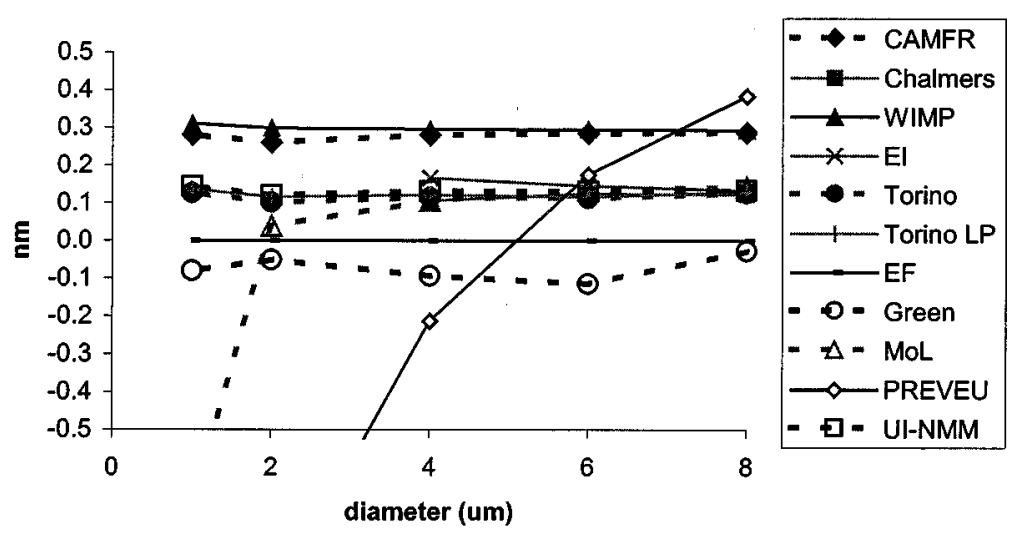

Fig. 7. Resonance wavelength difference with the EF model for the fundamental mode as a function of diameter for an antinode oxide.

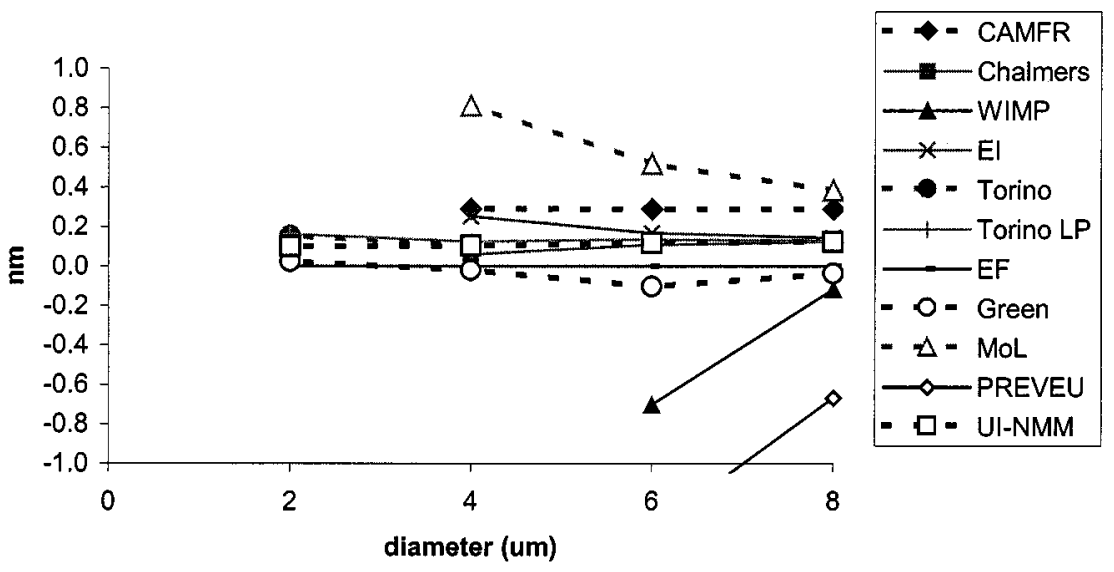

Fig. 8. Resonance wavelength difference with the EF model for the first-order mode as a function of diameter for an antinode oxide.
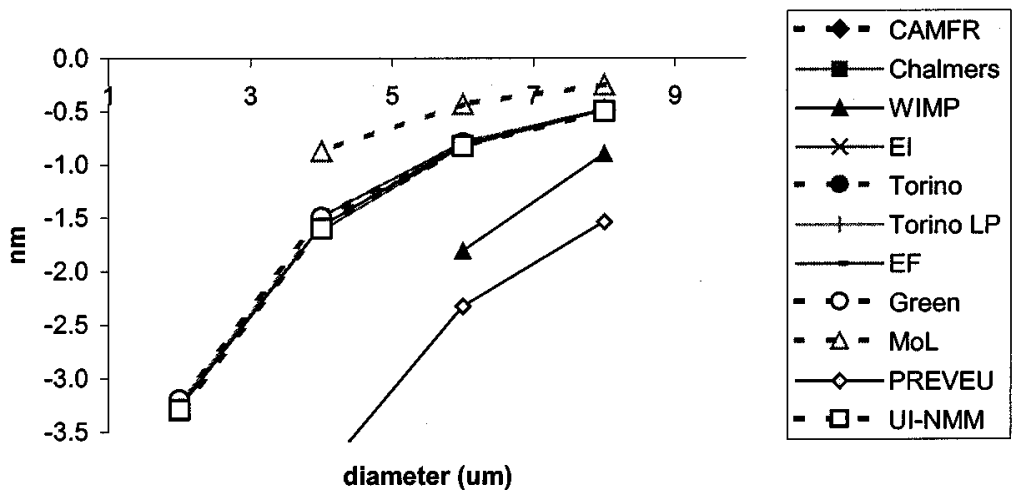

diameter (um)

Fig. 9. Difference in resonance wavelength between the first order mode and the fundamental mode as a function of device diameter.

quantity depends mostly on the aperture geometry (diameter, thickness, position) and not so much on the longitudinal profile of the structure [14]. Hence, it focuses on the 2-D portion of the modeling and eliminates the influence of the longitudinal profile (some models might suffer from the discretization along the longitudinal direction). This wavelength splitting is an important parameter: as an example, it is possible to extract the mode diameter from this value [28]. Fig. 9 shows that, apart from the MoL, WIMP, and PREVEU results, the spread in this wavelength difference is a lot smaller than the spread in the individual wavelengths (roughly $0.1 \mathrm{~nm}$ instead of $0.5 \mathrm{~nm}$ ).
Reducing the aperture size leads to a loss in confinement and higher thresholds, as can be seen in Fig. 10 (note the logarithmic scale). Once again, the differences between the models increase for smaller diameters, and vectorial models typically predict higher thresholds. Similar conclusions can be drawn from the relative threshold difference for the first-order mode in Fig. 11. The EF and EI models predict lower thresholds, because they do not take diffraction losses into account. The trend to show higher diffraction losses for smaller apertures is also much more pronounced for the vectorial models, and the relative spread between the vectorial models is, once again, much larger than be- 


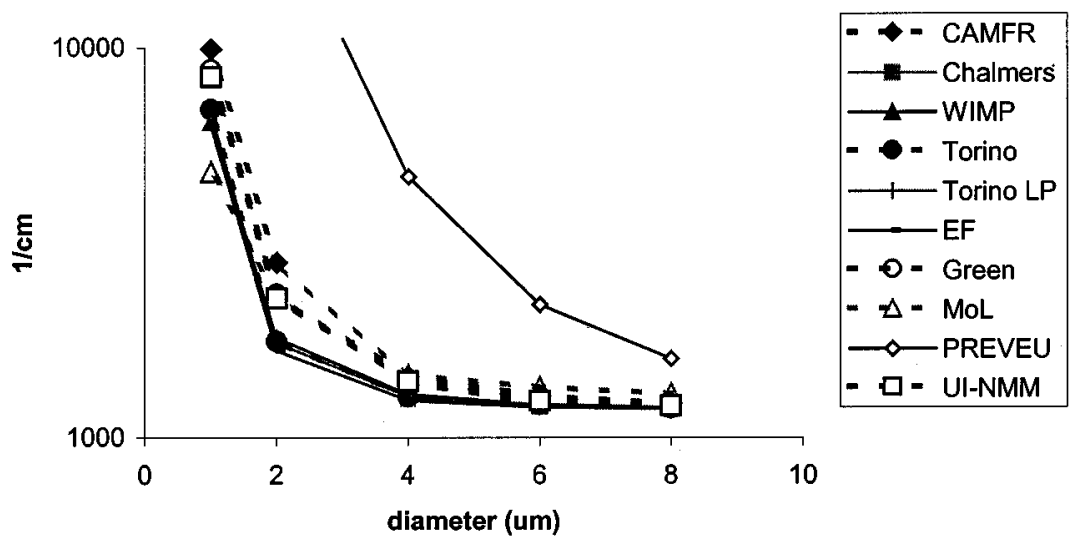

Fig. 10. Threshold material gain for the fundamental mode as a function of diameter for an antinode oxide. Note the logarithmic scale for the gain.

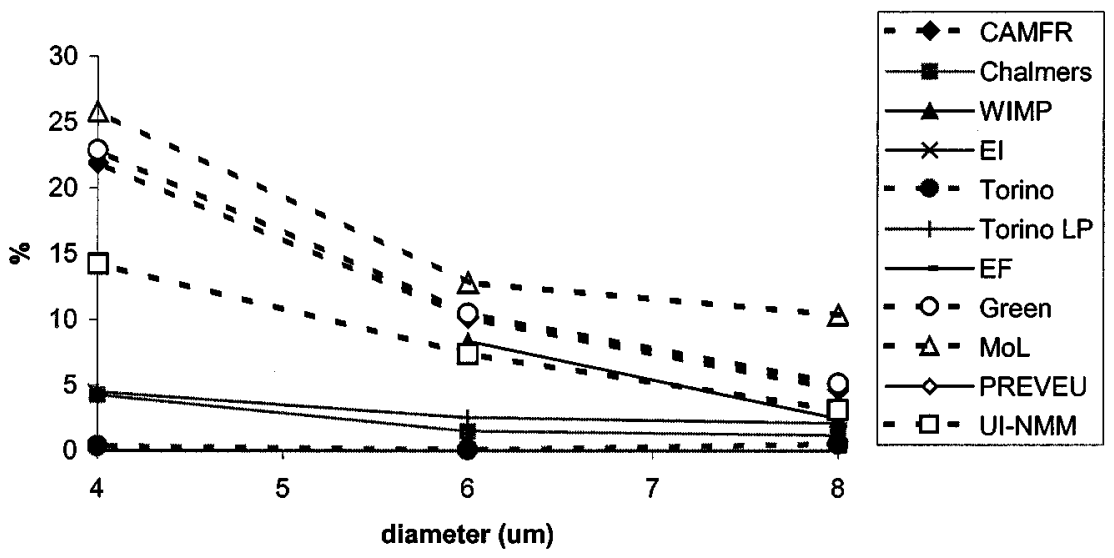

Fig. 11. Relative threshold material gain difference with the EF model for the first-order mode as a function of diameter for an antinode oxide.

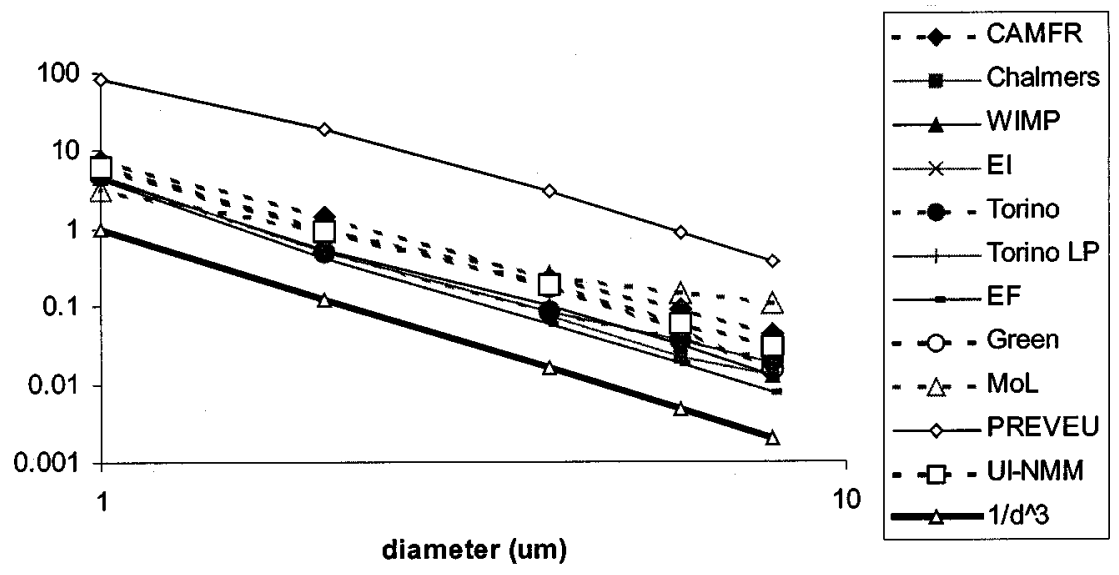

Fig. 12. Relative excess modal loss for the fundamental mode as a function of device diameter. Note the $\log -\log$ scale and the slope coefficient of -3 .

tween the scalar models. The WIMP model follows the trend of the vectorial models quite well, although it has some difficulty in modeling structures smaller than $6 \mu \mathrm{m}$ in diameter. The scalar and vectorial implementations of the Torino model give different results, but, strangely enough, the vectorial one predicts lower thresholds. This is explained by the fact that, in the scalar version of this model, the mirror reflectivity is a weighted average between the TE reflectivity and the slightly lower TM reflectivity. This results in an underestimation of the true $\mathrm{TE}_{01}$ reflectivity, and hence an overestimation of the threshold gain for the scalar model.

From the results presented so far, we can also argue more clearly that parasitic reflections are an important aspect in explaining the spread between the vectorial models. Looking again at Figs. 10 and 11, we can see that this spread is larger for smaller diameters and higher order modes, precisely the situ- 
TABLE II

Numerical Simulation Results For the Fundamental Mode in AN 8- $\mu \mathrm{m}$ VCSEL

\begin{tabular}{ccccccccccccc}
\hline \multicolumn{10}{c}{ Lambda $(\mathbf{n m})$} \\
pos & CAMFR & Chalmers & WIMP & EI & Torino & Torino LP & EF & Green & MoL & PREVEU UI-NMM \\
1 & 981.142 & 980.990 & 981.150 & 981.000 & 980.981 & 980.983 & 980.854 & 980.800 & 981.000 & 980.610 & 980.988 \\
2 & 980.884 & 980.730 & 980.880 & 980.730 & 980.722 & 980.725 & 980.597 & 980.500 & 980.800 & 980.500 & 980.730 \\
$\mathbf{3}$ & 980.615 & 980.460 & 980.610 & 980.460 & 980.450 & 980.453 & 980.327 & 980.200 & 980.550 & 980.390 & 980.460 \\
$\mathbf{4}$ & 980.366 & 980.200 & 980.360 & 980.210 & 980.200 & 980.202 & 980.079 & 980.000 & 980.320 & 980.330 & 980.212 \\
$\mathbf{5}$ & 980.214 & 980.050 & 980.220 & 980.060 & 980.053 & 980.057 & 979.927 & 979.900 & 980.070 & 980.310 & 980.061
\end{tabular}

Threshold material gain $(1 / \mathrm{cm})$

$\begin{array}{cccccccccccc}\text { pos } & \text { CAMFR } & \text { Chalmers } & \text { WIMP } & \text { EI } & \text { Torino } & \text { Torino LP } & \text { EF } & \text { Green } & \text { MoL } & \text { PREVEU UI-NMM } \\ 1 & 2690 & 2540 & 2530 & & 2498 & 2463 & 2441 & 2614 & 2660 & 1520 & 2486 \\ 2 & 1349 & 1270 & 1270 & & 1277 & 1277 & 1268 & 1294 & 1370 & 1526 & 1289 \\ 3 & 1247 & 1200 & 1199 & & 1204 & 1204 & 1193 & 1218 & 1330 & 1556 & 1217 \\ 4 & 1225 & 1190 & 1186 & & 1193 & 1193 & 1180 & 1205 & 1310 & 1584 & 1205 \\ 5 & 1224 & 1190 & 1190 & & 1197 & 1196 & 1184 & 1193 & 1300 & 1597 & 1209 \\ \end{array}$

TABLE III

NUMERICAL SimUlation RESUlts FOR THE FiRST-ORDER MODE IN AN 8- $\mu \mathrm{m}$ VCSEL

\begin{tabular}{|c|c|c|c|c|c|c|c|c|c|c|c|}
\hline \multirow[b]{2}{*}{ pos } & \multirow[b]{2}{*}{ CAMFR } & \multirow[b]{2}{*}{ Chalmers } & \multicolumn{4}{|c|}{ Lambda (nm) } & \multirow[b]{2}{*}{$\mathrm{EF}$} & \multirow[b]{2}{*}{ Green } & \multirow[b]{2}{*}{ MoL } & \multirow[b]{2}{*}{ PREVEU } & \multirow[b]{2}{*}{ UI-NMM } \\
\hline & & & WIMP & EI & Torino & Torino LP & & & & & \\
\hline 1 & 980.995 & & & & 980.714 & 980.716 & 980.549 & 980.500 & 980.890 & 979.500 & 980.692 \\
\hline 2 & 980.508 & 980.350 & & 980.350 & 980.344 & 980.347 & 980.219 & 980.100 & 980.650 & 979.280 & 980.349 \\
\hline 3 & 980.167 & 980.000 & 979.570 & 980.000 & 979.999 & 980.004 & 979.879 & 979.800 & 980.390 & 979.000 & 980.006 \\
\hline 4 & 979.890 & 979.730 & 979.430 & 979.730 & 979.718 & 979.723 & 979.602 & 979.500 & 980.150 & 978.830 & 979.727 \\
\hline 5 & 979.726 & 979.560 & 979.320 & 979.580 & 979.559 & 979.565 & 979.438 & 979.400 & 979.820 & 978.770 & 979.562 \\
\hline \multicolumn{12}{|c|}{ Threshold material gain $(1 / \mathrm{cm})$} \\
\hline pos & CAMFR & Chalmers & WIMP & EI & Torino & Torino LP & EF & Green & MoL & PREVEU & UI-NMM \\
\hline 1 & 9637 & & & & 13923 & 13826 & 14674 & 14918 & 7300 & 2055 & 14655 \\
\hline 2 & 1536 & 1500 & & & 1487 & 1498 & 1486 & 1539 & 1570 & 2341 & 1526 \\
\hline 3 & 1298 & 1260 & 1344 & & 1247 & 1263 & 1243 & 1308 & 1380 & 2525 & 1286 \\
\hline 4 & 1255 & 1210 & 1240 & & 1206 & 1224 & 1202 & 1270 & 1340 & 2623 & 1239 \\
\hline 5 & 1252 & 1210 & 1226 & & 1202 & 1221 & 1196 & 1257 & 1320 & 2666 & 1233 \\
\hline \multicolumn{12}{|c|}{ Modal stability (\%) } \\
\hline pos & CAMFR & Chalmers & WIMP & EI & Torino & Torino LP & EF & Green & MoL & PREVEU & UI-NMM \\
\hline 1 & 258.3 & & & & 82.1 & 461.3 & 501.1 & 470.8 & 174.4 & 35.2 & 489.5 \\
\hline 2 & 13.9 & 18.1 & & & 14.1 & 17.3 & 17.2 & 18.9 & 14.6 & 53.4 & 18.4 \\
\hline 3 & 4.1 & 5.0 & 12.1 & & 3.4 & 4.9 & 4.2 & 7.4 & 3.8 & 62.3 & 5.7 \\
\hline 4 & 2.4 & 1.7 & 4.6 & & 1.1 & 2.6 & 1.9 & 5.4 & 2.3 & 65.6 & 2.8 \\
\hline 5 & 2.3 & 1.7 & 3.0 & & 0.4 & 2.1 & 1.0 & 5.4 & 1.5 & 66.9 & 2.0 \\
\hline
\end{tabular}

ations where more radiation is propagating nonparaxially. For situations where we can assume the influence of parasitic reflections to be negligible, like for the fundamental mode in large devices, the spread between the models is very small.
Finally, we show in Fig. 12 the relative excess modal loss for the fundamental mode in the antinode oxide device. This quantity is defined as the relative difference $\left(g-g_{\text {planar }}\right) /$ $g_{\text {planar }}$ between the threshold material for the actual device 
TABLE IV

Numerical Simulation Results for the Fundamental Mode in AN ANTINODE OXIDE VCSEL

\begin{tabular}{|c|c|c|c|c|c|c|c|c|c|c|c|}
\hline \multicolumn{12}{|c|}{ Lambda (nm) } \\
\hline d & CAMFR & Chalmers & WIMP & EI & Torino & Torino LP & EF & Green & MoL & PREVEU & UI-NMM \\
\hline 8 & 980.214 & 980.050 & 980.220 & 980.060 & 980.053 & 980.057 & 979.927 & 979.900 & 980.070 & 980.310 & 980.061 \\
\hline 6 & 980.000 & 979.840 & 980.010 & 979.860 & 979.828 & 979.831 & 979.715 & 979.600 & 979.850 & 979.890 & 979.847 \\
\hline 4 & 979.475 & 979.300 & 979.490 & 979.360 & 979.309 & 979.315 & 979.194 & 979.100 & 979.300 & 978.980 & 979.326 \\
\hline 2 & 977.612 & & 977.650 & & 977.453 & 977.467 & 977.351 & 977.300 & 977.390 & 976.380 & 977.471 \\
\hline 1 & 975.061 & & 975.090 & & 974.906 & 974.916 & 974.780 & 974.700 & 974.070 & 972.710 & 974.924 \\
\hline \multicolumn{12}{|c|}{$\begin{array}{l}\text { Threshold material gain } \\
\qquad(1 / \mathrm{cm})\end{array}$} \\
\hline d & CAMFR & Chalmers & WIMP & EI & Torino & Torino LP & EF & Green & MoL & PREVEU & UI-NMM \\
\hline 8 & 1224 & 1190 & 1190 & & 1197 & 1196 & 1184 & 1193 & 1300 & 1597 & 1209 \\
\hline 6 & 1279 & 1200 & 1212 & & 1217 & 1217 & 1196 & 1231 & 1350 & 2205 & 1244 \\
\hline 4 & 1437 & 1260 & 1292 & & 1275 & 1274 & 1245 & 1386 & 1450 & 4673 & 1399 \\
\hline 2 & 2823 & & 1806 & & 1769 & 1756 & 1677 & 2340 & 2300 & 23954 & 2287 \\
\hline 1 & 9925 & & 6445 & & 6945 & 6818 & 6278 & 8780 & 4813 & 96465 & 8422 \\
\hline
\end{tabular}

TABLE V

Numerical Simulation Results for the First-Order Mode in AN ANTINODE OXIDE VCSEL

\begin{tabular}{|c|c|c|c|c|c|c|c|c|c|c|c|}
\hline \multicolumn{12}{|c|}{ Lambda (nm) } \\
\hline d & CAMFR & Chalmers & WIMP & EI & Torino & Torino LP & EF & Green & MoL & PREVEU & UI-NMM \\
\hline 8 & 979.726 & 979.560 & 979.320 & 979.580 & 979.559 & 979.565 & 979.438 & 979.400 & 979.820 & 978.770 & 979.562 \\
\hline 6 & 979.192 & 979.010 & 978.200 & 979.070 & 979.028 & 979.037 & 978.904 & 978.800 & 979.420 & 977.560 & 979.022 \\
\hline 4 & 977.910 & 977.680 & & 977.870 & 977.729 & 977.744 & 977.620 & 977.600 & 978.430 & 975.080 & 977.723 \\
\hline 2 & cut-off & cut-off & & cut-off & 974.231 & 974.237 & 974.08 & 974.100 & cut-off & 969.660 & 974.179 \\
\hline 1 & cut-off & cut-off & & cut-off & cut-off & cut-off & cut-off & cut-off & cut-off & cut-off & cut-off \\
\hline \multicolumn{12}{|c|}{$\begin{array}{l}\text { Threshold material gain } \\
\qquad(1 / \mathrm{cm})\end{array}$} \\
\hline d & CAMFR & Chalmers & WIMP & EI & Torino & Torino LP & EF & Green & MoL & PREVEU & UI-NMM \\
\hline 8 & 1252 & 1210 & 1225 & & 1202 & 1221 & 1196 & 1257 & 1320 & 2666 & 1233 \\
\hline 6 & 1357 & 1250 & 1335 & & 1233 & 1263 & 1232 & 1361 & 1390 & 4977 & 1323 \\
\hline 4 & 1696 & 1450 & & & 1396 & 1453 & 1391 & 1710 & 1750 & 14589 & 1589 \\
\hline 2 & cut-off & cut-off & & & 21765 & 21578 & 16990 & 20679 & cut-off & cut-off & 26879 \\
\hline 1 & cut-off & cut-off & & & cut-off & cut-off & cut-off & cut-off & cut-off & cut-off & cut-off \\
\hline \multicolumn{12}{|c|}{$\begin{array}{c}\text { Modal stability } \\
(\%)\end{array}$} \\
\hline d & CAMFR & Chalmers & WIMP & El & Torino & Torino LP & EF & Green & MoL & PREVEU & UI-NMM \\
\hline 8 & 2.3 & 1.7 & 2.9 & & 0.4 & 2.1 & 1.0 & 5.4 & 1.5 & $5 \quad 66.9$ & 2.0 \\
\hline 6 & 6.1 & 4.2 & 10.1 & & 1.3 & 3.8 & 3.0 & 10.5 & 3.0 & 125.7 & 6.4 \\
\hline 4 & 18.0 & 15.1 & & & 9.5 & 14.1 & 11.7 & 23.3 & 20.7 & 212.2 & 13.6 \\
\hline 2 & & & & & 1130.4 & 1128.8 & 913.1 & 783.7 & & & \\
\hline
\end{tabular}

and that of the planar device. This excess loss is mainly due to scattering losses at the oxide aperture. It has been observed that this quantity scales roughly as $1 / d^{3}$ [14], [29], which is confirmed by most of the models in the log-log plot of Fig. 12. Only the MoL curve tends to flatten a bit for large diameters. 


\section{CONCLUSION}

Clearly, there is no such thing as the "best" VCSEL model suited for all purposes, as there is a whole spectrum of models that all trade off accuracy and calculation time, which are both very important parameters for practical use. When one is only interested in device trends, paraxial EI-like models can give very good results at unparalleled speeds. For more accurate simulations, there are nonparaxial scalar models or hybrid models that combine some aspects of scalar and vectorial techniques. For full accuracy, vectorial models are required, but these typically have much longer computation times and also need to deal adequately with the problems of parasitic reflections to achieve their full potential.

Although all these models are mostly able to predict the correct qualitative trends, quantitative results are not always the same. As far as resonance wavelength is concerned, these differences are often below $0.5 \mathrm{~nm}$ and can be considered minor. Larger differences occur when studying smaller oxide apertures or higher order modes. For the resonance wavelength, there is no clear clustering between scalar and vectorial models, which indicates that the scalar approach yields reliable results. Much larger discrepancies are observed in the threshold material gain, where deviations of 10\%-30\% are not uncommon. Again, the spread increases for smaller diameters and higher order modes. In these cases, the EI and EF models break down and predict lower thresholds, because they are unable to take diffraction losses into account. Vectorial models do incorporate these effects, and they typically predict higher thresholds. However, the spread among the vectorial gain results is much larger than the spread among the scalar results, most likely due to numerical influences like parasitic reflections at the boundaries of the computational domain. This seems to be an important aspect to take into account when developing models aiming for the "next" level of high numerical accuracy.

\section{APPENDIX}

This appendix contains the numerical results of the simulations. The data for the 8- $\mu \mathrm{m}$ VCSEL as a function oxide position can be found in Tables II and III, for the fundamental and first order mode respectively. The results as a function of size for an antinode oxide are summarized in Tables IV and V.

\section{ACKNOWLEDGMENT}

J. -F. Seurin and S.L. Chuang would like to thank Professor W. C. Chew for helpful suggestions. M. Noble gratefully acknowledges the support of J.Lorenzo. K. Vaccaro, and S. Spaziani in providing the resources necessary to perform these calculations. He also thanks T. Nelson, P. Sotirelis, and J. Lott for their creative help and guidance.

\section{REFERENCES}

[1] D. Huffaker and D. Deppe, "Intracavity contacts for low-threshold oxide-confined vertical-cavity surface-emitting lasers," IEEE Photon. Technol. Lett., vol. 11, pp. 934-936, Aug. 1999.

[2] M. Grabherr, M. Miller, R. Jager, R. Michalzik, U. Martin, H. J. Unold, and K. J. Ebeling, "High-power VCSEL's: Single devices and densely packed 2-D-arrays," IEEE J. Select. Topics Quantum Electron., vol. 5, pp. 495-502, 1999 .
[3] S. Eitel, S. J. Fancey, H. P. Gauggel, K. H. Gulden, W. Bachtold, and M. R. Taghizadeh, "Highly uniform vertical-cavity surface-emitting lasers integrated with microlens arrays," IEEE Photon. Technol. Lett., vol. 12, pp. 459-461, May 2000.

[4] G. R. Hadley, "Effective index model for vertical-cavity surface-emitting lasers," Opt. Lett., vol. 20, pp. 1483-1485, 1995.

[5] J. A. Vukusic, H. Martinsson, J. S. Gustavsson, and A. Larsson, "Numerical optimization of the single fundamental mode output from a surface modified vertical cavity surface emitting laser," IEEE J. Quantum Electron., vol. 37, pp. 108-117, Jan. 2001.

[6] G. R. Hadley, K. L. Lear, M. E. Warren, K. D. Choquette, J. W. Scott, and S. W. Corzine, "Comprehensive numerical model for $\mathrm{cw}$ verticalcavity surface-emitting lasers," Phys. Simul. Optolectron. Devices III, vol. 2399, pp. 336-247, 1995.

[7] H. Wenzel and H. J. Wünsche, "The effective frequency method in the analysis of vertical-cavity surface-emitting lasers," IEEE J. Quantum Electron., vol. 33, pp. 1156-1162, 1997.

[8] S. Riyopoulos, D. Dialetis, J.-M. Inman, and A. Phillips, "Active cavity VCSEL eigenmodes with simple analytic representation," J. Opt. Soc. Amer. B, submitted for publication.

[9] S. Riyopoulos and D. Dialetis, "Radiation scattering by apertures in VCSEL cavities and its effects on mode structure," J. Opt. Soc. Amer. B, submitted for publication.

[10] G. P. Bava, P. Debernardi, and L. Fratta, "Three-dimensional model for vectorial fields in vertical-cavity surface-emitting lasers," Phys. Rev. A, vol. 63, p. 23816, 2001.

[11] C. Degen, I. Fischer, W. Elsaesser, G. P. Bava, P. Debernardi, L. Fratta, M. Brunner, R. Hovel, M. Moser, and K. Gulden, "Transverse modes in thermally detuned oxide confined vertical cavity surface emitting lasers," Phys. Rev. A, vol. 63, p. 23 817, 2001.

[12] Q.-H. Liu and W. C. Chew, "Numerical mode-matching method for the multiregion vertically stratified media," IEEE Trans. Antennas Propagat., vol. 38, pp. 498-506, Apr. 1990.

[13] B. Demeulenaere, P. Bienstman, B. Dhoedt, and R. G. Baets, "Detailed study of AIAs-oxidized apertures in VCSEL cavities for optimized modal performance," IEEE J. Quantum Electron., vol. 35, pp. 358-367, Mar. 1999.

[14] J.-F. P. Seurin, "Modeling of Vertical-Cavity Surface-Emitting Lasers and Comparison with Experiment," Ph.D. dissertation, Univ. Illinois, Urbana-Champaign, 2001.

[15] J.-F. P. Seurin, G. Liu, S. L. Chuang, L. M. F. Chirovsky, W. S. Hobson, and J. Lopata, "Modal competition in implant-apertured index-guided vertical-cavity surface-emitting lasers," Appl. Phys. Lett., vol. 77, no. 23, pp. 3686-3688, 2000.

[16] M. J. Noble, J. P. Loehr, and J. A. Lott, "Analysis of microcavity VCSEL lasing modes using a full-vector weighted index method," IEEE J. Quantum Electron., vol. 34, pp. 1890-1903, Oct. 1998.

[17] M. J. Noble, J.-H. Shin, K. D. Choquette, J. P. Loehr, J. A. Lott, and Y.-H. Lee, "Calculation and measurement of resonant-mode blueshifts in oxide-apertured VCSEL's," IEEE Photon. Technol. Lett., vol. 10, pp. 475-7, Apr. 1998.

[18] M. J. Noble, P. Sotirelis, T. Nelson, and J. A. Lott, "Calculation of threshold gain for oxide-apertured VCSEL's via the weighted index method with parasitic mode loss," IEEE J. Quantum Electron., submitted for publication.

[19] R. Pregla and E. Ahlers, "'New vector-BPM in cylindrical coordinates based on the method of lines'," in Integr. Photo. Resear. Tech. Dig., vol. 7, Dana Point, CA, 1995, pp. 23-25.

[20] E. Ahlers, S. F. Helfert, and R. Pregla, "'Modeling of VCSEL's by the method of lines,", in Proc. Integrated Phototograph Research Tech. Dig., vol. 6, Boston, MA, 1996, pp. 340-343.

[21] O. Conradi and R. Pregla, "'Considering azimuthal dependence in the numerical modeling of VCSELs'," Opt. Quantum Electron., vol. 32, pp. 759-767, 2000.

[22] S. F. Helfert and R. Pregla, "'Efficient analysis of periodic structures'," J. Lightwave Technol., vol. 16, pp. 1694-1702, Sept. 1998.

[23] B. Klein, L. F. Register, K. Hess, D. G. Deppe, and Q. Deng, "Self-consistent Green's function approach to the analysis of dielectrically apertured vertical-cavity surface-emitting lasers," Appl. Phys. Lett., vol. 73, no. 23, pp. 3324-3326, 1998.

[24] P. Bienstman and R. Baets, "Rigorous and efficient optical VCSEL model based on vectorial eigenmode expansion and perfectly match layers," IEEE J. Quantum Electron., submitted for publication.

[25] P. Bienstman, H. Derudder, R. Baets, F. Olyslager, and D. De Zutter, "Analysis of cylindrical waveguide discontinuities using vectorial eigenmodes and perfectly matched layers," IEEE Trans. Microwave Theory Tech., vol. 49, Feb. 2001. 
[26] P. Bienstman and R. Baets, "Optical modeling of photonic crystals and VCSEL's using eigenmode expansion and perfectly matched layers," Opt. Quantum Electron., vol. 33, no. 4/5, pp. 327-341, 2001, to be published.

[27] R. Kuszelewicz, COST 268 Modeling Exercise, CNET, Paris, France, 1998.

[28] L. A. Graham, D. L. Huffaker, and D. G. Deppe, "Spontaneous lifetime control in a native-oxide-apertured microcavity," Appl. Phys. Lett., vol. 74, no. 17 , pp. 2408-2410, 1999.

[29] E. R. Hegblom, N. M. Margalit, A. Fiore, and L. A. Coldren, "High-performance small vertical-cavity lasers: A comparison of measured improvements in optical and current confinement in devices using tapered apertures," IEEE J. Select. Topics Quantum Electron., vol. 5, pp. 553-560, Mar. 1999.

Peter Bienstman was born in Ghent, Belgium, in 1974. He received the degree in electrical engineering in 1997 the Ph.D. degree in 2001, both from Ghent University, Ghent, Belgium.

His research interests include the modeling of optical structures, notably resonant-cavity LEDs, VCSELs, and photonic crystal structures. He has published several papers and has one patent application pending.

Dr. Bienstman a student member of the IEEE Lasers and Electro-Optics Society.

Roel Baets received the degree in electrical engineering from Ghent University, Ghent, Belgium, in 1980, the M.Sc. degree in electrical engineering from Stanford University, Stanford, CA, in 1981, and the Ph.D. degree from Ghent University in 1984

Since 1981, he has been with the Department of Information Technology (INTEC) of Ghent University, where he has been a Professor since 1989. He has worked in the field of III-V devices for optoelectronic systems. With approximately 200 publications and conference papers, he has made contributions to the design and fabrication of semiconductor laser diodes, passive guided-wave devices, PICs, and microoptic components.

Dr. Baets is a member of the Optical Society of America, the IEEE Lasers and Electro-Optics Society, SPIE, and the Flemish Engineers Association.

Josip Vukusic was born in Gothenburg, Sweden, in 1972. He received the M.Sc. degree in engineering physics from Chalmers University of Technology, Gothenburg, Sweden, in 1996, where he is currently working toward the Ph.D. degree.

In 1997, he joined the Photonics Laboratory at University of Technology. His research is mainly centered around surface-emitting devices, particularly VCSELS.

Anders Larsson received the M.S. and Ph.D. degrees in electrical engineering from Chalmers University of Technology, Gothenburg, Sweden, in 1982 and 1987, respectively.

In 1991, he joined the faculty at Chalmers University of Technology where be became a Professor in 1994. From 1984 to 1985, he was with the Department of Applied Physics, California Institute of Technology, Pasadena, and from 1988 to 1991, he was with the Jet Propulsion Laboratory, Pasadena. His background is in the area of quantum-well materials and devices for optical communication, optical information processing, and infrared detection. Currently, his research is focused on surface-emitting lasers (vertical-cavity surface-emitting lasers and grating coupled surface-emitting lasers), diffractive integrated optics, and new gain materials for semiconductor lasers.

Michael J. Noble received the B.S. degree from Rensselaer Polytechnic Institute, Troy, NY, and the M.S. and Ph.D. degrees from the Air Force Institute of Technology, all in electrical engineering.

Currently a Major, he has been an officer in the United States Air Force since 1989. He is presently affiliated with the Air Force Research Laboratory, Sensors Directorate, Hanscom AFB, MA, where he performs theoretical research on microcavity VCSELs, multi-spectral quantum-well infrared photodetectors, and laser radar systems. He is the author of more than one refereed journal article.
Marcel Brunner was born in Rüti, Switzerland, in 1969. He received the Dipl.Phys. degree from the Centre National de Recherche Scientifique, Grenoble, France, in 1995, and the Ph.D. (Dr.Sc.) degree from the Ecole Polytechnique Federale de Lausanne, Lausanne, Switzerland, in 2000.

Prior to his doctoral work, he joined the Optoelectronic Devices section of the Centre Suisse d'Electronique et de Microtechnique, Zürich, Switzerland. He is currently with Avalon Photonics Ltd., Zürich, Switzerland, a manufacturer of short-wavelength vertical-cavity surface-emitting laser arrays.

Karlheinz Gulden received the Diploma and Ph.D. degrees from the University of Erlangen-Nurnberg, Germany, in 1990 and 1994, respectively.

He has over 13 years experience in designing high-speed semiconductor devices, crystal growth, fabrication, and testing. From 1994 to 1997, he was responsible for VCSEL development at the Paul Scherrer Institute, Zürich, Switzerland, addressing applications in sensors and optical interconnects. In 1997, he joined CSEM as Section Head of Optoelectronic Devices. In 2000, he co-founded Avalon Photonics Ltd., Zürich, Switzerland, and has been leading the company since that time as CEO. He has written and co-written more than 50 articles on VCSELs and optoelectronic switches and holds two patents with a further two pending.

Pierluigi Debernardi was born in Casale Monferrato, Italy, in 1963. He received the degree in electronics engineering from the Politecnico di Torino, Torino, Italy, in 1987.

Since 1989, he has been with IRITI, the Politecnico di Torin, an Italian National Reseach Council Institute. His interests are mainly in the field of the modeling of semiconductor materials and devices for optoelectronic applications. In particular, he studied III-V quantum wells, computing their optical response with Coulomb effects included on the basis of the second quantization approach. He was involved in ridge waveguide laser simulation, analysis of optical bistable behavior of multiple-quantum-well devices, and four-wave mixing in semiconductor oscillators and amplifiers. Now he works mainly on microcavity lasers, photonic bandgap structures, and VCSELs, computing their electromagnetic properties to evaluate the spontaneous emission factor, threshold, and noise behavior.

Laura Fratta was born in Parma, Italy, in 1971. She received the degree in physics from the University of Milan, Milan, Italy, in 1996. She is currently working toward the Ph.D. degree in electronic engineering at the Politecnico of Torino, Turin, Italy, where she is involved in studying the properties of verticalcavity surface-emitting lasers (VCSELs).

In particular, she has worked on polarization properties, especially in presence of noise, linewidth performances and a 3-D model for modes in VCSELs.

Gian Paolo Bava was born in Varallo Sesia, Italy, in 1937. He received the degree in electrical engineering from Politecnico di Torino, Torino, Italy, in 1961.

Since then, he has been with the Instituto di Elettronica e Telecomunicazioni (now Dipartimento di Elettronica), where he has been a Full Professor of Microwave Photonics since 1976. His research activity has been concerned successively with travelling wave tubes for satellite applications, microwave semicondutor devices (Mesfet, Gunn effect, etc.), and amplifiers, both regarding simulation and characterization, integrated optics, and optoelectronics mainly with reference to parametric oscillators and semiconductor lasers. Recently, his interests have been mainly in the field of the modeling of semiconductor materials and devices. At present, he is involved in the modeling of VCSELs.

Hans Wenzel received the Diploma and Doctoral degrees in physics from Humboldt-University, Berlin, Germany, in 1986 and 1991, respectively. His thesis was concerned with the electro-optical modeling of semiconductor lasers.

From 1991 to 1994, he was involved in a research project on the three-dimensional simulation of distribued feedback lasers. In 1994, he joined the Ferdinand-Braun-Institut für Höchstfrequenztechnik, Berlin, Germany, were he is engaged in the development of high-power semiconductor lasers. His research interests include the analysis, modeling, and simulation of a large variety of optoelectronic devices. 
Benjamin Klein received the B.S. and M.S. degrees in electrical engineering from the University of Wisconsin-Madison in 1994 and 1995, respectively, and the Ph.D. degree from the University of Illinois at Urbana-Champaign in 2000.

He is currently working at the National Institute of Standards and Technology, Boulder, $\mathrm{CO}$.

Olaf Conradi was born in Adenau, Germany, in 1970. He received the B.S. degree in electrical engineering (Dipl.Ing.) from the Fachhochschule Köln, Germany, in 1995 and the M.S. degree in electrical engineering (Dipl.Ing. degree) from the University Siegen, Germany, in 1996.

Since 1996, he has been with the FernUniversität, Hagen, Germany, as a Research Assistant, where he is involved in the analysis of vertical-cavity laser diodes.

Reinhold Pregla (M'76-SM'83-F'99) received the Dipl.-Ing degree and the Dr.-Ing degree in electrical engineering from the Technische Universität Braunschweig, Federal Republic of Germany, in 1963 and 1966, respectively.

Since 1975, he has been a Full Professor in Electrical Engineering at the Fern Universität (a university for distance study), Hagen, Germany. His fields of investigation include microwave and millimeter-wave integrated circuits, field theory, antennas and integrated optics, and photonics. He is the inventor of the Method of Lines for applications in these fields.

In 1999, he was elected as IEEE Fellow for his contributions to the analysis, modeling, and design of microwave and optical components.

Spilios A. Riyopoulos received the B.Sc. degree in physics from the University of Athens, Greece, in 1977 and the Ph.D. degree in physics from the University of Maryland, College Park, in 1983.

He has broad experience in theoretical and computational physics. His earlier work on nonlinear plasma theory, ranging from coherent and stochastic wave-particle interactions to nonlinear magneto-hydrodynamic effects, was applied to coherent microwave sources, (electron cyclotron masers, free electron lasers) and tokamak RF-heating and stability. Recently, his work has turned to optoelectronics, focusing on semiconductor laser theory and simulation. Current topics of interest are cavity-mode structure, mode competition, and long-range interactions in VCSEL arrays.
Jean-François P. Seurin (S'00) was born in Rodez, France, in 1971. He received the degree in electrical engineering (Diplôme d'Ingénieur) from the Ecole Nationale Supérieure des Télécommunications (ENST), Paris, France, in 1995, and the M.S. and Ph.D. degrees in electrical engineering from the University of Illinois at Urbana-Champaign in 1996 and 2001, respectively. His doctoral research focused on the theoretical and experimental investigation of index-guided VCSELs for optical communication applications.

His main research interests are in the fields of electromagnetics, numerical modeling, optoelectronics, and fiber optics. He is currently with Princeton Optronics, Mercerville, NJ, a manufacturer of long-wavelength optoelectronic devices.

Shun Lien Chuang received the B.S. degree in electrical engineering from National Taiwan University, Taiwan, R.O.C., in 1976 and the M.S., E.E., and Ph.D. degrees in electrical engineering from Massachusetts Institute of Technology, Cambridge, in 1980, 1981, and 1983, respectively.

In 1983, he joined the Department of Electrical and Computer Engineering at the University of Illinois at Urbana-Champaign, where he is currently a Professor. He was a Resident Visitor at AT\&T Bell Laboratories, Holmdel, NJ, in 1989 and a consultant at Bellcore and Polaroid in 1991. He was a Senior Visiting Professor (Sabbatical Chair) at SONY Research Center in 1995 and an Invited Professor at NTT Basic Research Laboratories in 1997. He was also a visitor at NASA Ames Research Center in the summer of 1999. He is conducting research on strained quantum-well semiconductor lasers, electroabsorption modulators, fiber optic sensors, and femtosecond nonlinear optics for terahertz radiation. He is the author of Physics of Optoelectronic Devices (New York: Wiley, 1995). He has published around 200 journal and conference papers and has given many invited talks at conferences and institutions.

Dr. Chuang is an Associate Editor of the IEEE Journal OF QUANTUM ELECTRONICS. He was a Feature Editor for a special issue in the Journal of Optical Society of America B on Terahertz Generation, Physics and Applications in 1994. He is a Fellow of the Optical Society of America and a member of the American Physical Society. He has been cited many times for Excellence in Teaching at the University of Illinois. He received the Andersen Consulting Award for excellence in advising in 1994 and was selected as an Associate a the Center for Advanced Study at the University of Illinois in 1995. He was also awarded a Fellowship from the Japan Society for the Promotion of Science to visit the University of Tokyo in 1996. 